\title{
An Approach to the Dimension Theory of Continuous Geometry from the Standpoint of Boolean Valued Analysis
}

By

\author{
Hirokazu NisHIMURA*
}

\begin{abstract}
Recently Boolean valued analysis (i.e., analysis based on Boolean valued set theory) has been extensively studied by G. Takeuti [14-16]. The main purpose of this paper is to show, by using this technique, that any continuous geometry can be viewed as an irreducible continuous geometry in its center valued set theory. This makes the transition from irreducible continuous geometries to reducible ones automatic.
\end{abstract}

\section{§1. Introduction}

Boolean valued set theory was introduced in 1966 by D. Scott and $R$. Solovay as a reformulation of P. J. Cohen's forcing techniques, which have been applied successfully to many significant independence results of set theory. Recently G. Takeuti [14-16] has applied this generalized set theory to various areas of analysis like Hilbert spaces, von Neumann algebras, harmonic analysis, etc. This approach reduces many formidable concepts and intractible theories in analysis to much easier and more familiar ones (e.g., self-adjoint operators as real numbers in a Boolean valued sense). The significance of Boolean algebras in analysis has been recognized at latest since M. H. Stone's classical work in the 1930s, but it is Boolean valued analysis that succeeded in showing its significance in its full power. As a slogan, we may say that wherever there is a complete Boolean algebra, there must be a Boolean valued analysis on it!

By the way, J. von Neumann [10] introduced continuous geometry in the middle of the 1930 s as a lattice-theoretical generalization of projective geometry, whose subject had just turned out to be no other than irreducible

Received January 23, 1984.

* Research Institute for Mathematical Sciences, Kyoto University, Kyoto 606, Japan. 
finite-dimensional complemented modular lattices from a lattice-theoretical viewpoint. He succeeded in developing a dimension theory for irreducible continuous geometries, but failed to generalize it to the reducible case. This difficulty was overcome by some Japanese mathematicians, say, T. Iwamura [3] and Y. Kawada et al. [6] in the middle of the 1940s.

The main purpose of this paper is to show that a possibly reducible continuous geometry is no other than an irreducible continuous geometry in some Boolean valued sense. This viewpoint makes the transition from irreducible to reducible continuous geometries automatic. In particular, it shows very clearly why the dimensions of reducible continuous geometries should be represented by real valued continuous functions on some appropriate topological space, while those of irreducible continuous geometries are represented simply by real numbers. After reviewing Boolean valued analysis and continuous geometry in Sections 2 and 3 respectively, we give the details of our approach in Section 4.

Last but not least, this paper was inspired most by G. Takeuti's [16] Boolean valued approach to von Neumann algebras and S. Maeda's [9] treatment of dimension theory. The results of this paper could be generalized, e.g., to the framework of S. Maeda [9] without difficulty*, but here we prefer concentrating on one of the most typical cases to pursuing their full generalizations.

\section{§ 2. Boolean Valued Analysis}

Let $\mathscr{B}$ be a complete Boolean algebra. We define $V_{\alpha}^{(\mathscr{B})}$ by transfinite induction on ordinal $\alpha$ as follows:

$$
\begin{aligned}
& V_{0}^{(\mathscr{B})}=\phi, \\
& V_{a}^{(\mathscr{B})}=\left\{u \mid u: \mathscr{D}(u) \rightarrow \mathscr{B} \text { and } \mathscr{D}(u) \subseteq \bigcup_{\xi<\infty} V_{\xi}^{(\mathscr{B})}\right\}
\end{aligned}
$$

Then the Boolean valued universe $V^{(\mathscr{B})}$ of Scott-Solovay is defined as follows:

$V^{(\mathscr{B})}=\bigcup_{\alpha \in \mathrm{On}} V_{\alpha}^{(\mathcal{B})}$, where On is the class of all ordinal numbers.

$V^{(\mathscr{B})}$ can be considered to be a Boolean valued model of set theory by defining $\llbracket u \in v \rrbracket$ and $\llbracket u=v \rrbracket$ for $u, v \in V^{(\mathscr{B})}$ with the following properties

\footnotetext{
* Such a generalization has been attempted by K. Eda (Tsukuba University).
} 
(1)

$$
\begin{aligned}
& \llbracket u \in v \rrbracket=\sup _{y \in \mathscr{D}(v)}(v(y) \wedge \llbracket u=y \rrbracket), \\
& \llbracket u=v \rrbracket=\inf _{x \in \mathscr{D}(u)}(u(x) \Rightarrow \llbracket x \in v \rrbracket) \wedge \inf _{y \in \mathscr{D}(v)}(v(y) \Rightarrow \llbracket y \in u \rrbracket),
\end{aligned}
$$

and by assigning a Boolean value $[\varphi]$ to each formula $\varphi$ without free variables inductively as follows:

$$
\begin{aligned}
& \llbracket \neg \varphi \rrbracket=\urcorner \llbracket \varphi \rrbracket=1-\llbracket \varphi \rrbracket, \\
& \llbracket \varphi_{1} \vee \varphi_{2} \rrbracket=\llbracket \varphi_{1} \rrbracket \vee \llbracket \varphi_{2} \rrbracket, \\
& \llbracket \varphi_{1} \wedge \varphi_{2} \rrbracket=\llbracket \varphi_{1} \rrbracket \wedge \llbracket \varphi_{2} \rrbracket, \\
& \llbracket \forall x \varphi(x) \rrbracket=\inf _{u \in V^{(\mathscr{B})}} \llbracket \varphi(u) \rrbracket, \\
& \llbracket \exists x \varphi(x) \rrbracket=\sup _{u \in V^{(\mathscr{B})}} \llbracket \varphi(u) \rrbracket .
\end{aligned}
$$

The following theorem is fundamental to Boolean valued analysis.

Theorem 2.1. If $\varphi$ is a theorem of $Z F C$, then so is $\llbracket \varphi \rrbracket=1$.

Now we present several elementary properties of $V^{(\mathscr{B})}$ without proofs.

\section{Proposition 2.2.}

$$
\begin{aligned}
& \llbracket \exists x \in u \varphi(x) \rrbracket=\sup _{x \in \mathscr{D}(u)}(u(x) \wedge \llbracket \varphi(x) \rrbracket), \\
& \llbracket \forall x \in u \varphi(x) \rrbracket=\inf _{x \in \mathscr{D}(u)}(u(x) \Rightarrow \llbracket \varphi(x) \rrbracket) .
\end{aligned}
$$

The class $V$ of all sets can be embedded into $V^{(\mathscr{B})}$ by transfinite induction as follows.

$$
\check{y}=\{\langle\grave{x}, 1\rangle \mid x \in y\} \quad \text { for } y \in V \text {. }
$$

Proposition 2.3. For $x, y \in V$,

$$
\begin{aligned}
& \llbracket \check{x} \in \check{y} \rrbracket= \begin{cases}1 & \text { if } x \in y \\
0 & \text { otherwise. }\end{cases} \\
& \llbracket \check{x}=\check{y} \rrbracket= \begin{cases}1 & \text { if } x=y \\
0 & \text { otherwise. }\end{cases}
\end{aligned}
$$

A subset $\left\{b_{\alpha}\right\}$ of $\mathcal{B}$ is called a partition of unity if $\sup _{\alpha} b_{\infty}=1$ and $b_{\alpha} \wedge b_{\beta}$ $=0$ for any $\alpha \neq \beta$. Given a partition of unity $\left\{b_{\alpha}\right\}$ and a subset $\left\{u_{\alpha}\right\}$ of $V^{(\mathscr{B})}$, 
it can be proved easily that:

Proposition 2.4. There exists an element $u$ of $V^{(\mathscr{B})}$ such that $\llbracket u=u_{\infty} \rrbracket \geqq b_{\infty}$ for any $\alpha$. Furthermore this $u$ is determined uniquely in the sense that $\llbracket u=v \rrbracket=$ 1 for any $v \in V^{(\mathscr{B})}$ with the above property.

The above $u$ is denoted by $\sum_{\infty} u_{\infty} b_{\infty}$ or $u_{\omega_{1}} b_{\alpha_{1}}+\cdots+u_{\omega_{n}} b_{\omega_{n}}$ if $\left\{b_{\alpha}\right\}$ is a finite set. Then we have

Proposition 2.5. $\llbracket \varphi\left(\sum_{\infty} u_{\infty} b_{\omega}\right) \rrbracket=\sup \left(\llbracket \varphi\left(u_{\infty}\right) \rrbracket \wedge b_{\infty}\right)$.

The techniques of partitions of unity gives the following two propositions.

Proposition 2.6 (The Maximum Principle). Let $\varphi$ be a formula. Then there exists a $u \in V^{(\mathcal{B})}$ such that $\llbracket \varphi(u) \rrbracket=\llbracket \exists x \varphi(x) \rrbracket$.

Proposition 2.7. Let $\varphi(x)$ be a formula with only $x$ as a free variable and $\llbracket \varphi(u) \rrbracket=1$ for some $u \in V^{(\mathscr{B})}$. Then

$$
\begin{aligned}
& \llbracket \forall x(\varphi(x) \Rightarrow \psi(x))]=\inf _{\llbracket \varphi(u) \rrbracket=1}[\psi(u) \rrbracket, \\
& \left.\llbracket \exists x(\varphi(x) \wedge \psi(x)) \rrbracket=\sup _{\llbracket \varphi(u) \rrbracket=1} \llbracket \psi(u)\right] .
\end{aligned}
$$

We define the interpretation $X^{(\mathscr{B})}$ of $X=\{x \mid \varphi(x)\}$ with respect to $V^{(\mathscr{B})}$ to be $\left\{u \in V^{(\mathscr{B})} \mid[\varphi(u) \rrbracket=1\}\right.$, assuming that it is not empty. For technical convenience, if $X$ is a set, then $X^{(\mathscr{B})}$ is usually considered to be a set by choosing a representative from an equivalence class $\left\{v \in V^{(\mathscr{B})} \mid \llbracket u=v \rrbracket=1\right\}$. Then, by Proposition 2.7 , we have $X^{(\mathscr{B})} \times\{1\} \in V^{(\mathscr{B})}$ and $\left.\llbracket X=X^{(\mathscr{B})} \times\{1\}\right]=1$.

Let $D \subseteq V^{(\mathscr{B})}$. A function $g: D \rightarrow V^{(\mathscr{B})}$ is called extensional if $\llbracket d=d^{\prime} \rrbracket \leqq$ $\llbracket g(d)=g\left(d^{\prime}\right) \rrbracket$ for any $d, d^{\prime} \in D . \quad$ A $\mathscr{B}$-valued set $u \in V^{(\mathscr{B})}$ is said to be definite if

$$
u(d)=1 \text { for any } d \in \mathscr{D}(u)
$$

Then we have the following characterization theorem of extensional maps.

Theorem 2.8. Let $u, v \in V^{(\mathscr{B})}$ be definite and $D=\mathscr{D}(u)$. Then there is a bijective correspondence between $f \in V^{(\mathscr{B})}$ satisfying $\llbracket f: u \rightarrow v \rrbracket=1$ and extensional maps $\varphi: D \rightarrow v^{(\mathscr{B})}$, where $v^{(\mathscr{B})}=\{u \mid \llbracket u \in v \rrbracket=1\}^{*}$. The correspondence is given by the relation $\llbracket f(d)=\varphi(d) \rrbracket=1$ for any $d \in D$.

\footnotetext{
* This is also considered to be a set by the above method.
} 
We conclude this section by considering what the real numbers in $V^{(\mathscr{B})}$ look like. First of all, we note that the set of all natural numbers in $V^{(\mathscr{B})}$ is $\check{\omega}$ and the sel of all rational numbers in $V^{(\mathscr{B})}$ is $\check{\mathrm{Q}}$. We define a real number to be the lower half line without the endpoint of a Dedekind cut. Thus " $a$ is a real number" is expressed formally by

$$
\begin{aligned}
& a \subseteq \mathbb{Q} \wedge \exists s \in \mathbb{Q}(s \in a) \wedge \exists s \in \mathbb{Q}(s \in a) \wedge \\
& \forall s \in \mathbb{Q}(s \in a \Leftrightarrow \exists t \in \mathbb{Q}(s<t \wedge t \in a)) .
\end{aligned}
$$

Let $\Omega$ be the Stone space of $\mathscr{B}$. Then $\mathscr{B}$ is isomorphic to the clopen subsets of $\Omega$, assigning a clopen set $E(b)$ to each $b \in \mathscr{B}$. Since $\mathscr{B}$ is complete, the closure of every open subset of $\Omega$ is open (cf. Ogasawara [11; Chapter 2, §4]). In particular, every regular open subset of $\Omega$ is clopen. Therefore, if we denote the Borel $\sigma$-field of $\Omega$ and the $\sigma$-ideal of meager Borel subsets of $\Omega$ by $\mathcal{S}$ and $\mathcal{G}$ respectively, then $\mathscr{B}$ is $\sigma$-isomorphic to $\mathcal{S} / \mathcal{G}$ (cf. Takeuti and Zaring $[13 ; \S 3$, Theorem 3.14]). For any $S$ in $\mathcal{S}$, we denote by $[S]$ the corresponding element in $\mathscr{B}$ by this isomorphism. By using a similar argument to Takeuti [14; Chapter $2, \S 2]$, we can establish a correspondence between $\mathbb{R}^{(\mathscr{B})}$ and the set $B_{R}(\Omega)$ of all real Borel functions on $\Omega$. The correspondence is given by the relation $\llbracket \check{r} \in u \rrbracket=[\{x \in \Omega \mid r<f(x)\}]$. By identifying real Borel functions on $\Omega$ which are equal except meager sets, this correspondence can be made bijective. Since every real Borel function on $\Omega$ is equal to some real continuous function on $\Omega$ except meager sets (cf. Ogasawara 111; Chapter 2 , $\S 4]$ ) and no two different real continuous functions on $\Omega$ can not be equal even except meager sets (cf. Takeuti and Zaring [13; §3, Theorem 3.23]), we have

Theorem 2.9. There is a bijective correspondence between $\mathbb{R}^{(\mathscr{B})}$ and $C_{R}(\Omega)$, where $C_{\mathbb{R}}(\Omega)$ denotes the set of all real continuous functions on $\Omega$.

\section{§3. Comtinuous Geometry}

Let $L$ be a lattice. Then for any directed set $D$, a family $\left\{a_{\delta}\right\}_{\delta \in D}$ of $L$ is called a monotone increasing system (a monotone decreasing system) if $\delta_{1} \leqq \delta_{2}$ implies $a_{\delta_{1}} \leqq a_{\delta_{2}}\left(a_{\delta_{2}} \leqq a_{\delta_{1}}\right.$ resp.). A continuous geometry is a complete complemented modular lattice $L$ satisfying the following continuity axioms:

$$
\sup _{\delta \in D}\left(a_{\delta} \wedge b\right)=\left(\sup _{\delta \in D} a_{\delta}\right) \wedge b
$$

for any monotone increasing system $\left\{a_{\delta}\right\}_{\delta \in D}$ of $L$; 


$$
\inf _{\delta \in D}\left(a_{\delta} \vee b\right)=\left(\inf _{\delta \in D} a_{\delta}\right) \vee b
$$

for any monotone decreasing system $\left\{a_{\delta}\right\}_{\delta \in D}$ of $L$.

Throughout the rest of this section, $L$ is assumed to be a continuous geometry unless stated to the contrary. An element $z$ of $L$ is called central if $(a \vee b) \wedge z=(a \wedge z) \vee(b \wedge z)$ for any $a, b \in L$. The set of all central elements of $L$, which is known to be a complete Boolean algebra and to be closed under sup and inf, is called the center of $L$ and denoted by $Z(L)$. Obviously $1,0 \in$ $Z(L)$. If $Z(L)=\{1,0\}$, then $L$ is called irreducible. Otherwise $L$ is called reducible. For any element $a \in L$, there is a least element $z \in Z(L)$ with respect to the property $a \leqq z$, which is called the central envelope of $a$ and denoted by $e(a)$.

Two elements $a, b$ of $L$ are called perspective and written $a \sim b$ if they have a common complement, i.e., $a \vee x=b \vee x=1$ and $a \wedge x=b \wedge x=0$ for some $x \in L$. It is known that perspectivity satisfies the transitive law in continuous geometry and so is an equivalence relation. We write $a<b$ or $b>a$ if $a \sim b_{1}<b$ for some $b_{1} \in L$. Then we have the following characterization theorem of irreducibility in terms of $\sim$ and $<$.

Theorem 3.1 (cf. Maeda [7; Chap. 4, §2]). A continuous geometry L is irreducible iff one of the three relations $a<b, a \sim b, b<a$ holds for any $a, b \in L$.

The fundamental theorem of the dimension theory for irreducible continuous geometries goes as follows:

Theorem 3.2 (cf. Maeda [7: Chap. 5, §2]). Let $L$ be an irreducible continuous geometry. Then there exists a unique real valued function $f$ on $L$ such that:

$$
\begin{aligned}
& 0 \leqq f(a) \leqq 1 \text { for any } a \in L ; \\
& f(0)=0 \text { and } f(1)=1 ; \\
& f(a \vee b)+f(a \wedge b)=f(a)+f(b) \text { for any } a, b \in L .
\end{aligned}
$$

\section{§4. The Construction of $\tilde{L}$ from $L$}

Let $L$ be an arbitrary continuous geometry. Let $\mathcal{B}=Z(L)$. The main purpose of this section is to construct a derived continuous geometry $\tilde{L}$ in $V^{(\mathscr{B})}$ and then to establish some correspondences between $L$ and $\tilde{L}$, which will enable us to derive the dimension theory of reducible continuous geometries from that of irreducible continuous geometries without much effort. 
Proposition 4.1. For any $a, b \in L$, there is a largest central element $z$ of $L$ with respect to the property $a \wedge z=b \wedge z$.

Proof. We write $Z(a, b)$ for $\{z \in Z(L) \mid a \wedge z=b \wedge z\}$. If $z \in Z(a, b)$ and $z \geqq z^{\prime} \in Z(L)$ then $a \wedge z^{\prime}=a \wedge z \wedge z^{\prime}=b \wedge z \wedge z^{\prime}=b \wedge z^{\prime}$ and so $z^{\prime} \in Z(a, b)$. If $z_{1}, z_{2} \in Z(a, b)$, then $a \wedge\left(z_{1} \vee z_{2}\right)=\left(a \wedge z_{1}\right) \vee\left(a \wedge z_{2}\right)=\left(b \wedge z_{1}\right) \vee\left(b \wedge z_{2}\right)=b \wedge\left(z_{1} \vee\right.$ $z_{2}$ ) and so $z_{1} \vee z_{2} \in Z(a, b)$. Therefore $Z(a, b)$ is an ideal. Thus, using the continuity axiom of continuous geometry, we have $(\sup Z(a, b)) \wedge a=\sup _{z \in Z(a, b)}$ $(z \wedge a)=\sup _{z \in Z(a, b)}(z \wedge b)=(\sup Z(a, b)) \wedge b$, which implies sup $Z(a, b) \in Z(a, b)$. This is the desired element.

For any $a, b \in L$, we write $e(a, b)$ for the element whose existence was established in the above proposition.

Now we define a binary relation $R$ on $\check{L}$ in $V^{(\mathscr{B})}$ as follows:

$$
R=\left\{\left\langle\langle a, b\rangle^{\vee}, e(a, b)\right\rangle \mid a, b \in L\right\} .
$$

Then we have:

Proposition 4.2. $\llbracket R$ is an equivalence relation on $\stackrel{L}{L} \rrbracket=1$.

Proof. This follows readily from the fact that for any $a, b, c \in L$,

$$
\begin{aligned}
& R\left(\langle a, a\rangle^{\vee}\right)=1 \\
& R\left(\langle a, b\rangle^{\vee}\right)=R\left(\langle b, a\rangle^{\vee}\right) ; \\
& R\left(\langle a, b\rangle^{\vee}\right) \wedge R\left(\langle b, c\rangle^{\vee}\right) \leqq R\left(\langle a, c\rangle^{\vee}\right) .
\end{aligned}
$$

Therefore we can consider the quotient set of $\check{L}$ with respect to the equivalence relation $R$ in $V^{(\mathscr{B})}$, which is denoted by $\tilde{L}$. For any $a \in L$, the equivalence class of $\stackrel{v}{a}$ with respect to $R$ in $V^{(\mathscr{B})}$ is denoted by $\tilde{a}$. Then it is easy to see that:

Proposition 4.3. $\llbracket \tilde{a}=\tilde{b} \rrbracket=e(a, b)$ for any $a, b \in L . \quad$ In particular, $\llbracket \tilde{a}=\tilde{b} \rrbracket=$ 1 iff $a=b$.

Proof. $\llbracket \tilde{a}=\tilde{b} \rrbracket=\llbracket \grave{a} R \tilde{b} \rrbracket=e(a, b)$.

Now we consider partitions of unity in $V^{(\mathscr{B})}$.

Proposition 4.4. For any partition of unity $\left\{z_{\infty}\right\}$ and any family $\left\{a_{\infty}\right\}$ of $L$ with the same index set, we have

$$
\sum \tilde{a}_{\alpha} z_{\alpha}=\left(\sup _{\alpha}\left(a_{\alpha} \wedge z_{\alpha}\right)\right)^{\sim} .
$$


Proof. It is easy to see, by dint of the continuity axiom, that

$$
\left(\sup _{a}\left(a_{a b} \wedge z_{a s}\right)\right) \wedge z_{\infty}=a_{a s} \wedge z_{a b}
$$

Therefore, $z_{\alpha} \leqq e\left(\sup _{\omega}\left(a_{\omega} \wedge z_{\alpha}\right), a_{\alpha}\right)=\llbracket\left(\sup _{\infty}\left(a_{a s} \wedge z_{\alpha}\right)\right)^{\sim}=\tilde{a}_{\alpha \rrbracket} \rrbracket$ by Proposition 4.3.

Corollary 4.5. $(\tilde{L})^{(\mathscr{B})}=\{\tilde{a} \mid a \in L\}$.

Now we would like to make $\tilde{L}$ a lattice in $V^{(\mathscr{B})}$.

Proposition 4.6. For any $a_{1}, a_{2}, b_{1}, b_{2} \in L$,

$$
\begin{aligned}
& \llbracket \tilde{a}_{1}=\tilde{a}_{2} \rrbracket \wedge \llbracket \tilde{b}_{1}=\tilde{b}_{2} \rrbracket \leqq \llbracket\left(a_{1} \vee b_{1}\right)^{\sim}=\left(a_{2} \vee b_{2}\right)^{\sim} \rrbracket, \\
& \llbracket \tilde{a}_{1}=\tilde{a}_{2} \rrbracket \wedge \llbracket \tilde{b}_{1}=\tilde{b}_{2} \rrbracket \leqq \llbracket\left(a_{1} \wedge b_{1}\right)^{\sim}=\left(a_{2} \wedge b_{2}\right)^{\sim} \rrbracket .
\end{aligned}
$$

Proof. We deal only with (1). If $a_{1} \wedge z=a_{2} \wedge z$ and $b_{1} \wedge z=b_{2} \wedge z$ for some $z \in Z(L)$, then $\left(a_{1} \vee b_{1}\right) \wedge z=\left(a_{1} \wedge z\right) \vee\left(b_{1} \wedge z\right)=\left(a_{2} \wedge z\right) \vee\left(b_{2} \wedge z\right)=\left(a_{2} \vee b_{2}\right) \wedge z$ Thus (1) follows readily by dint of Proposition 4.3.

By dint of Theorem 2.8 and the above proposition, we can safely define lattice operations $\vee$ and $\wedge$ on $\tilde{L}$ in $V^{(\mathscr{B})}$ as follows:

$$
\begin{aligned}
& \tilde{a} \vee \tilde{b}=(a \vee b)^{\sim}, \\
& \tilde{a} \wedge \tilde{b}=(a \wedge b)^{\sim} .
\end{aligned}
$$

By dint of Corollary 4.3, it is almost trivial to see that:

Theorem 4.7. $\llbracket \tilde{L}$ is a continuous geometry $\rrbracket=1$.

We denote $\{\tilde{z} \mid z \in Z(L)\} \times\{1\}$ by $\tilde{Z}$. Then it is also trivial to see that:

Theorem 4.8. $\llbracket$ The center of $\tilde{L}$ is $\tilde{Z} \rrbracket=1$.

Theorem 4.9. $\llbracket \tilde{L}$ is an irreducible continuous geometry $\rrbracket=1$.

Proof. For $z \in Z(L)$, we have

$$
\llbracket \tilde{z}=\tilde{1} \vee \tilde{z}=\tilde{0} \rrbracket=\llbracket \tilde{z}=\tilde{1} \rrbracket \vee \llbracket \tilde{z}=\tilde{0} \rrbracket=z \vee \neg z=1 .
$$

Then the theorem follows from Theorems 4.7 and 4.8.

Now any theorem in irreducible continuous geometries can be transferred automatically to that on $\widetilde{L}$ in $V^{(\mathscr{B})}$. As an application of this principle, we can obtain the fundamental theorem of the dimension theory for continuous geometries in its general form from that for irreducible continuous geometries. 
Theorem 4.10. For any continuous geometry $L$, there exists a unique function $f$ from $L$ to $C_{\boldsymbol{R}}(\Omega)$ (the value of $f$ at $a \in L$ is denoted by $f_{a}$ rather than $f(a)$ ) satisfying the following properties:

(1) $0 \leqq f_{a}(\mathfrak{p}) \leqq 1$ for any $\mathfrak{p} \in \Omega$;

(2) For any central element $z \in L, f_{z}(\mathfrak{p})= \begin{cases}1 & \text { if } \mathfrak{p} \in E(z) \\ 0 & \text { otherwise }\end{cases}$

(3) $f_{a \vee b}(\mathfrak{p})+f_{a \wedge b}(\mathfrak{p})=f_{a}(\mathfrak{p})+f_{b}(\mathfrak{p})$ for any $\mathfrak{p} \in \Omega$.

Proof. By using Theorem 2.8, we can easily see that there is a bijective correspondence between real valued functions on $\tilde{L}$ in $V^{(\mathscr{B})}$ and functions $f$ from $L$ to $C_{R}(\Omega)$ satisfying the following property $(*)$.

$\left(^{*}\right)$ For any $a, b \in L$ and any $z \in Z(L)$, if $a \wedge z=b \wedge z$, then $f_{a}$ and $f_{b}$ coincide on $E(z)$.

Since the functions $f$ with the three properties of the theorem satisfies the property $\left({ }^{*}\right)$, the theorem follows readily from Theorems 3.2 and 4.9.

We give some further correspondences between $L$ and $\tilde{L}$.

Proposition 4。目目. For any $a \in L, e(a)=\llbracket \tilde{a} \neq \tilde{0} \rrbracket$.

Proof. $e(a)=\urcorner e(a, 0)=\urcorner \llbracket \tilde{a}=\tilde{0} \rrbracket=\llbracket \tilde{a} \neq \tilde{0} \rrbracket$.

For any $a, b \in L$, we write $a \quad b$ iff for any $z \in Z(L)$, either $z \wedge a<z \wedge b$ or $z \wedge a=z \wedge b=0$.

Proposition 4.12. For any $a, b \in L, a \quad b$ iff $\llbracket \tilde{a}>\tilde{b} \rrbracket=0$.

For any $a, b \in L$, we write $a \ll b$ iff for any $z \in Z(L)$, either $z \wedge a<z \wedge b$ or $z \wedge a=z \wedge b=0$.

Proposition 4.12. For any $a, b \in L, a \ll b$ iff. $[\tilde{a}>\tilde{b}]]=0$.

Proof. By Theorems 3.1 and 4.9, $\llbracket \tilde{a}>\tilde{b} \vee \tilde{a} \sim \tilde{b} \vee \tilde{a}<\tilde{b} \rrbracket=1$. Therefore $[\tilde{a}>\tilde{b} \rrbracket=0$ iff $[\tilde{a} \sim \tilde{b} \vee \tilde{a}<\tilde{b}]=1$ iff $a \ll b$.

Theorem 4.13. For any continuous geometry $L, L$ is of type $k(1 \leqq k \leqq \infty)$ iff $\tilde{L}$ is of type $k$ in $V^{(\mathscr{B})}$.

To conclude this section, we consider an intriguing problem whether every irreducible continuous geometry in $V^{(\mathscr{B})}$ is of the form $\tilde{L}$ for some appropriate continuous geometry $L$. Our answer is positive. 
Theorem 4.14. Let $\mathscr{B}$ be an arbitrary complete Boolean algebra. Then any irreducible continuous geometry in $V^{(\mathcal{B})}$ is, up to isomorphism, of the form $\tilde{L}$ for some continuous geometry $L$ whose center is $\mathscr{B}$.

Proof. Let $\hat{L}$ be an irreducible continuous geometry in $V^{(\mathscr{B})}$. Let $L=$ $\left\{u \in V^{(\mathscr{B})} \mid[\lfloor u \in \hat{L} \rrbracket=1\}\right.$. It is easy to see that $L$ is a continuous geometry with the derived lattice structure from $\hat{L}$. Now we would like to determine the center of $L$. It is easy to see that $a \in L$ is central iff $\llbracket a$ is central $\rrbracket=1$. But since $\hat{L}$ is irreducible in $V^{(\mathscr{B})}$, $\llbracket a$ is central $\rrbracket=\llbracket a=1 \vee a=0 \rrbracket$. Therefore, there is a bijective correspondence between $Z(L)$ and $\mathcal{B}$, assigning to $z \in Z(L) \llbracket z=1 \rrbracket$ $\in \mathscr{B}$ and conversely assigning to $x \in \mathscr{B}(1 x+0\urcorner x) \in Z(L)$. Thus we can identify $Z(L)$ with $\mathscr{B}$. Now it remains to show that $\llbracket a=b \rrbracket=e(a, b)$ for any $a$, $b \in L . \quad$ But, for any $x \in \mathscr{B}$, we have $\llbracket(1 x+0\urcorner x) \wedge a=(1 x+0\urcorner x) \wedge b \rrbracket=(\llbracket 1 \wedge$ $a=1 \wedge b \rrbracket \wedge x) \vee([0 \wedge a=0 \wedge b \rrbracket) \wedge \neg x)=(\llbracket a=b \rrbracket \wedge x) \vee \neg x$. Therefore $(1 x+$ $\left.0^{-} x\right) \leqq e(a, b)$ iff $x \leqq \llbracket a=b \rrbracket$. This completes the proof.

Theorems 4.9 and 4.14 show that continuous geometries in $V$ whose center is $\mathcal{B}$ and irreducible continuous geometries in $V^{(\mathscr{B})}$ are the same things from different viewpoints. However the transition from one viewpoint to the other is fruitful enough to yield seemingly formidable concepts and theorems from simpler ones automatically.

\section{References}

[1] Halperin, I., On the transitivity of perspectivity in continuous geometries, Trans. Amer. Math. Soc., 44 (1938), 537-562.

[2] — Dimensionality in reducible geometries, Annals of Math., 40 (1939), 581599.

[ 3 ] Iwamura, T., On continuous geometries I, Japan J. Math., 19 (1944), 57-71.

[ 4 ] -, On continuous geometries II, J. Math. Soc. Japan, 2 (1950), 148-164.

[5] Jech, T., Set theory, Academic Press, New York, 1978.

[6] Kawada, Y. et al., Bemerkungen zum vorangehenden Arbeit von Herrn T. Iwamura, Japan J. Math., 19 (1944), 73-79.

[ 7 ] Maeda, F., Continuous geometry (in Japanese), Iwanami, Tokyo, 1952.

[ 8$]^{*} \longrightarrow$, Kontinuierliche Geometrien, Springer, New York, 1958.

[9] Maeda, S., Dimension functions on certain general lattices, J. Sci. Hiroshima Univ., A19 (1955), 211-237.

[10]**von Neumann, J., Continuous Geometry, Princeton Univ. Press, Princeton, 1960.

[11] Ogasawara, T., Lattice theory II (in Japanese), Iwanami, Tokyo, 1948.

[12] Ozawa, M., Boolean valued interpretation of Hilbert space theory, J. Math. Soc. Japan, 35 (1983), 609-627.

* This is a German translation of Maeda [7].

** This is his Princeton lecture notes during 1935-1937 edited by I. Halperin after his death. 
[13] Takeuti, G. and Zaring, W.M., Axiomatic set theory, Springer, New York, 1973.

[14] Takeuti, G., Two applications of logic to mathematics, Iwanami and Princeton Univ. Press, Tokyo and Princeton, 1978.

[15] - A transfer principle in harmonic analysis, J. Symbolic Logic, 44 (1979), $417-440$.

[16] - von Neumann algebras and Boolean valued analysis, J. Math. Soc. Japan, 35 (1983), 1-21. 
